\title{
Caracterización química de la vidriera de Sant Pere i Sant Jaume (segundo cuarto del s. XIV, Monestir de Pedralbes, Barcelona)
}

\author{
D.GIMENO (1), M.PUGÈS (2) \\ (1) Dept. Geoquímica, Petrologia i Prospecció Geològica, Fac. Geologia, Universitat de Barcelona, 08028 - Barcelona. \\ (2) Servei d'Arqueologia del Museu d'Història de la Ciutat, Ajuntament de Barcelona, Zona Franca. Sector C. C/F, 22
}

\begin{abstract}
Se han caracterizado químicamente diferentes tipos de vidrios arquitectónicos originales de la iglesia del Monestir de Pedralbes, Barcelona (realizados hacia 1330). Esta nota expone los resultados obtenidos mediante el empleo de microsonda electrónica en vidrios de varios colores (blanco, verde, rosado, azul, amarillo, rojo) de la vidriera de Sant Pere y Sant Jaume. Corresponden esencialmente a vidrios sódicos (dentro del rango 13-19\% de $\mathrm{Na}_{2} \mathrm{O}$ ) de coloración y composición homogénea, con elementos que actúan como colorante (p.e., $\mathrm{CuO}$ en rango 1.6-6 \% en los vidrios verdes). El vidrio rojo, potásico, es una excepción notable $\left(16.5 \% \mathrm{~K}_{2} \mathrm{O}, 14 \% \mathrm{CaO}\right)$, tecnológicamente diferente (vidrio "plaqué" blanco con una intercalación roja de composición análoga pero sensiblemente más rica en $\mathrm{CuO}$ ).

Los vidrios sódicos no aparecen substancialmente dañados, los vidrios potásicos presentan corrosiones (moderadas) y pérdida parcial de transparencia. El predominio en esta vidriera de vidrios sódicos (inusuales para la época por lo que conocemos) ha permitido una excelente conservación. La composición química es una característica que permite diagnosticar precozmente el estado de deterioro y evaluar los costes de restauración en una vidriera, independientemente de los recubrimientos, pátinas y morteros que ésta presente.
\end{abstract}

Palabras clave: vidrieras medievales, composición, vidrios sódicos coloreados, vidrio rubí potásico

Chemical characterization of the stained glass window of Sant Pere and Sant Jaume (second quarter of the XIV century, Monestir de Pedralbes, Barcelona)

Several architectural medieval stained glass (1330 a.C.) from Pedralbes monastery's church (Barcelona, Spain) have been characterised chemically. This note explains the results obtained with EMPA, representative of bulk chemistry of several coloured glasses (white, green, pink, blue, yellow, red) coming from the Sant Pere and Sant Jaume stained-glass window. Most of them are Na-rich (13-19\% $\left.\mathrm{Na}_{2} \mathrm{O}\right)$ homogeneous in colour and composition, and show minor variations in bulk chemistry related with colour (i.e., $\mathrm{CuO}^{2}$ in the 1.6-6 \% range in green glass). Singularly, red glass is $\mathrm{K}-\mathrm{rich}(16.5 \% \mathrm{~K} \mathrm{O}, 14 \%$ $\mathrm{CaO}$ ), and was elaborated in a way technologically more sophisticated (it is a plaqué glass, with a red Cu-rich layer sandwiched between a white transparent $\mathrm{Cu}$-poor glass similar in composition).

While Na-rich glasses are mostly unaltered, K-rich ones show moderate corrosion and partial loss of transparency. Dominance of Na-rich glasses in the stained glass windows (rare at that time, if we consider the present state of the art) allowed an excellent conservation. Despite of the presence of mortar covering, patina, etc., bulk chemistry of the glass shows its value as a diagnostic tool in an early evaluation of the state of decay of an stained glass window.

Keywords: medieval stained glass, chemical composition, coloured sodium glass, potassium rubi glass.

\section{INTRODUCCIÓN}

A pesar del importante patrimonio de vidrieras arquitectónicas históricas existente en Europa (del que España no es una excepción), el conocimiento de las características composicionales de estas vidrieras sigue siendo relativamente escaso, y los datos disponibles aparecen bastante dispersos en la bibliografía especializada (1, 2, etc.). Este trabajo expone los primeros resultados referidos a la composición de las vidrieras de la iglesia del Monestir de Pedralbes en Barcelona, concretamente de las vidrieras de Sant Pere y Sant Jaume (3). Estas vidrieras fueron realizadas hacia 1330 y se han conservado en su disposición original, frecuentemente incluso con su emplomado original.

Los motivos de este desconocimiento sobre la composición de las vidrieras son variados. En primer lugar las vidrie- ras han sido consideradas tradicionalmente como un arte menor (p.e., con respecto a la pintura), con lo que los escasos recursos de todo tipo disponibles ante un patrimonio artístico ingente que transmitir a nuestros sucesores han llegado en poca medida a su mantenimiento y restauración. Por su ubicación arquitectónica los controles sobre su degradación, y las facilidades para su estudio y muestreo han sido limitadas tradicionalmente a los trabajos de restauración que, por motivos económicos y de mera funcionalidad (la mayor parte de este patrimonio aparece en lugares de culto abiertos al público) se han desarrollado muy espaciada y excepcionalmente en el tiempo. De hecho, la que es posiblemente la recopilación analítica más importante sobre vidrio antiguo (1) procede en buena medida de obras de arte que han sido descontextualizadas y recogidas en un museo. 
El estudio de algunas de las características físico-químicas de los vidrios históricos permite alcanzar diferentes objetivos: a/ establecer cual es su composición; b/ qué transformaciones han sufrido, en términos de procesos y productos; y c/ establecer estrategias de intervención. Estos objetivos han de entenderse secuencialmente, ya que las transformaciones son función directa de la composición y cualquier tipo de intervención que ignore composición y transformaciones implica riesgos serios en un material que como el vidrio es intrínsecamente metaestable.

En este trabajo nos hemos limitado al primero de estos objetivos, es decir caracterizar químicamente un conjunto de vidrios históricos que nos han llegado relativamente bien conservados. Por lo que se refiere estrictamente a los problemas analíticos que implica este tipo de materiales, podemos señalar dos como principales: la escasez de material disponible en cada muestra, y su riqueza en elementos alcalinos. La primera viene condicionada por la misma naturaleza de la muestra que, en la medida de lo posible debe ser mantenida en la vidriera o restituida a ésta en el caso de fragmentos rotos accidentalmente. Sin embargo, si queremos caracterizar correctamente la composición química del vidrio por un método fiable, económico y accesible a muchos equipos de trabajo es inevitable emplear una técnica destructiva. En este caso por lo tanto el compromiso reside en desarrollar un protocolo analítico fiable, útil a los efectos requeridos, y que implique sacrificar la mínima cantidad posible de vidrio. En todo caso, no está de más el señalar que, p.e. en el caso de vidrieras medievales, es muy raro el encontrar vidrios intactos por lo que, siendo perfectamente respetuosos en el proceso de restauración, parece fácil poder obtener las muestras necesarias, en especial cuando se trata de intervenciones sobre grandes superficies.

\section{CARACTERÍSTICAS MACROSCÓPICAS Y PETROGRÁFICAS DE LOS VIDRIOS}

En general podemos concluir que las vidrieras de Sant Pere y Sant Jaume presentan un buen estado de conservación (véase fotos 1 y 2), tanto en lo que se refiere a vidrios como a grisallas. Los vidrios presentan un espesor variable, en general entre 1.5 y $3.5 \mathrm{~mm}$ (moda $2 \mathrm{~mm}$ aproximadamente). Desde el punto de vista de la factura son frecuentes las burbujas ocluídas (véase foto 2) con forma de elipsoide de revolución, de hasta varios mm de diámetro, y cuerdas térmicas producto del estiramiento de las anteriores. Todos los vidrios estudiados, con la excepción del rojo rubí que es de tipo plaqué y será comentado independientemente a continuación, presentan un carácter homogéneo y una coloración uniforme; macroscópicamente no presentan evidencias notables de alteración.

El vidrio rojo presenta en general una factura más fina, espesores frecuentemente menores y menor cantidad de burbujas ocluídas. Por lo que se refiere al color, se trata de un vidrio blanco en el que el color aparece concentrado en una de las caras, en un estrato rojo que a su vez aparece recubierto de nuevo por una delgada lámina de vidrio blanco. En el detalle microscópico se observa que el estrato rojo está en realidad constituido por una fina sucesión de laminillas coloreadas alternadas con el vidrio blanco que actúa de soporte. El estado de conservación de este vidrio rojo, aún siendo bueno, es peor que el del resto, presentando leves veladuras con la consiguiente pérdida de transparencia y frecuente pérdida o disminución parcial del delgado estrato blanco que protege el color. El estudio (microscopio electrónico de barrido con

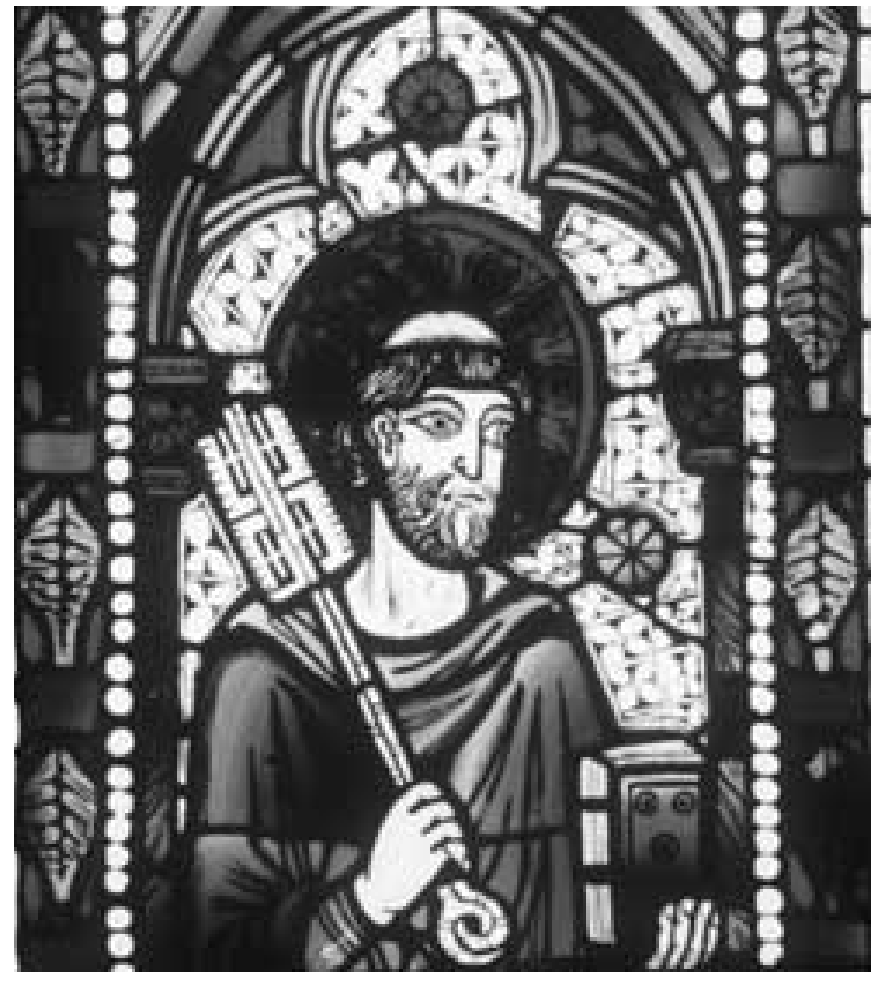

Figura 1. Vidriera de Sant Pere, iglesia del Monestir de Pedralbes, Barcelona.

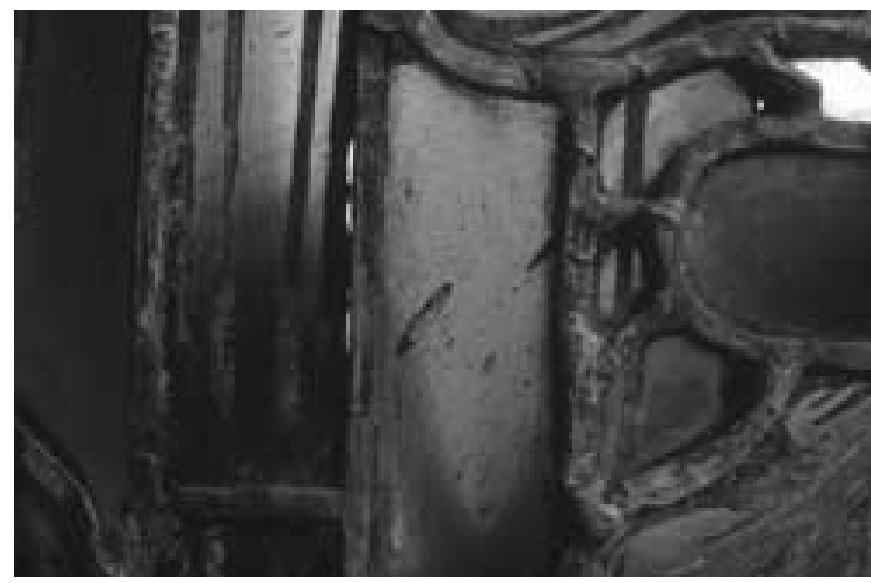

Figura 2. Detalle de la vidriera de Sant Jaume, iglesia del Monestir de Pedralbes, Barcelona. Nótese la presencia de emplomado original entre vidrios de diferentes colores y, en el del centro, una prominente burbuja deformada durante el proceso de soplado y estirado del vidrio. 
microanalizador, difracción de rayos X) confirma la presencia de sulfatos (yeso, mucho más raramente singenita) en la superficie de las veladuras y localmente en núcleos de alteración que se presentan como leves hinchamientos circulares o elongados en la superficie del vidrio. Además se pueden distinguir picaduras (de diámetro inferior en general a $0.5 \mathrm{~mm}$ ), atribuibles a ataque por microorganismos. Todas estas características tienen una correlación con la composición del vidrio, como se verá a continuación.

La totalidad de los vidrios han sido estudiados en lámina delgada con el concurso del microscopio petrográfico. La lámina fue previamente preparada introduciendo el vidrio en disco portante confeccionado con una resina epoxy y realizando un corte perpendicular a la superficie de los vidrios. Los vidrios empleados proceden de fragmentos recuperados de dimensiones reducidas, no reintegrables a la vidriera original durante el proceso de reemplomado, y ha sido catalogada su procedencia durante el proceso de documentación de cada uno de los pasos realizados en la restauración. El montaje en la resina ha permitido su orientación y corte perpendicular a la superficie, lo que permite reconocer petrográficamente y mediante la microsonda las posibles variaciones de composición y textura relacionadas con la alteración de los vidrios, desde el exterior al interior de éstos, garantizando por lo tanto acceder en todo caso a las porciones internas inalteradas de éste. Previamente fueron muestreados y analizados por difracción de rayos $\mathrm{X}$ los posibles productos cristalinos de la alteración superficial de los vidrios, o en su caso las pátinas superpuestas a éstos.

En general se observa una ausencia generalizada de distorsiones en la transmisión de la luz, escaso desarrollo de microfracturación y ausencia de frentes evidentes de gelificación o de desvitrificación; igualmente, ausencia de elementos refractarios (infundidos). En vidrios de color verde se ha detectado la presencia de desarrollo incipiente de haces fibroso-radiales (aparentemente, fases cristalinas de sílice) y el crecimiento de un microlito esquelético atribuible a un feldespato de tipo albita-oligoclasa. En vidrios naturales este tipo de microlitos se forman por fenómenos de sobreenfriamiento de magmas (4) o directamente por cristalización precoz ya en el estado sólido (5).

\section{PARTE EXPERIMENTAL}

Los análisis de vidrios históricos de Pedralbes han sido realizados mediante toda una serie de técnicas que incluyen espectroscopía de fluorescencia de rayos X (FRX) sobre matriz diluida en fundente (tetraborato de litio) y directamente sobre el vidrio plano original, microscopía electrónica de barrido (MEB) con microanalizador basado en dispersión de energías de rayos $\mathrm{X}$ (EDS en el acrónimo anglosajón), microsonda electrónica (MSE), espectrometría de absorción atómica (EAA), etc.; se han encaminado tanto a la caracterización de la matriz base del vidrio como a la identificación específica de los elementos o sales minerales colorantes empleados. Estos procedimientos analíticos son en parte complementarios y en parte repetitivos, y lógicamente forman únicamente parte de un estudio comparativo (que será objeto de una publicación específica) encaminado a la obtención de una metodología básica de trabajo mediante el empleo de la MSE que ha permitido, por una parte, que podamos afrontar el estudio de los componentes principales de un vidrio histórico a partir de fragmentos de 0,5 grs o menores ( $10 \mathrm{~mm} \times 5 \mathrm{~mm}$ x espesor del vidrio, o menor), y que estos análisis sean tan fiables p.e. como los obtenidos por FRX mediante dilución en perla de tetraborato de litio (considerados un estándard de calidad de análisis en muestra de roca, y por lo tanto en obsidianas y otros vidrios naturales).

Se exponen en este trabajo únicamente los resultados obtenidos mediante el concurso de la MSE; las principales ventajas de esta técnica radican en su carácter multielemental (que permite determinar tanto la totalidad de los elementos mayoritarios que constituyen el vidrio como, indirectamente, controlar la validez del análisis ya que éste debe cerrar en valores próximos al 100\%), el escaso volumen de muestra requerido y su accesibilidad en términos tanto económicos como de laboratorios en funcionamiento en nuestro país.

Por otra parte, el principal problema relacionado con la determinación de la composición de los vidrios medievales reside en su elevado contenido en elementos alcalinos que han sido incorporados a la pasta base del vidrio en tanto que fundentes. Los elementos alcalinos, y en particular el $\mathrm{Na}$, presentan notables problemas en su determinación mediante los procedimientos analíticos físico-químicos más comúnmente disponibles en nuestros laboratorios, de tal manera que se suelen analizar separadamente por métodos que implican solubilización total de la muestra (p.e. espectroscopía de absorción atómica, EAA). A título de ejemplo podemos indicar que en vidrios naturales semejantes a los aquí estudiados (p.e. riolitas) hemos detectado a lo largo de 10 años subestimaciones sistemáticas del $\mathrm{Na}$ cuando aparece por debajo de cantidades del orden del 2-2.5 \% de $\mathrm{Na}_{2} \mathrm{O}$ en las determinaciones mediante FRX respecto a EAA.

En el caso específico de la MSE, la superficie metalizada (con grafito) de la muestra sufre un bombardeo de electrones que hace que resulte calentada puntualmente durante el período de incidencia del haz a temperaturas de hasta $700^{\circ} \mathrm{C}$ (dependiendo de las condiciones instrumentales y de la máquina empleada), siendo igualmente sometida a un campo eléctrico, de modo que los elementos alcalinos (6) como el $\mathrm{K}$, en especial el $\mathrm{Na}$ literalmente migran a lo largo de la muestra tras un brevísimo periodo de incubación, huyendo del punto irradiado y dando tanto subestimaciones sistemáticas de los elementos alcalinos como análisis que cierran en sumatorios de valores muy inferiores al $100 \%$. Este efecto se produce tanto en vidrios (naturales o artificiales) como en minerales y compuestos que presenten los elementos alcalinos implicados en enlaces cristaloquímicos débiles. Las soluciones que se han propuesto a este problema son variadas, e incluyen variaciones notables de las condiciones instrumentales, p.e. con reducción notable del tiempo de adquisición, desplazamiento programado de la lámina vítrea problema durante el periodo de irradiación, congelamiento previo de la muestra, etc. Las muestras estudiadas en este trabajo han sido analizadas mediante un protocolo contrastado previamente en el estudio de vidrios naturales volcánicos diferenciados (del rango de composición riolita, traquita, fonolita y dacita) que incluye un porcentaje notable de rocas peralcalinas (7), es decir, las que presentan un rango composicional más semejante a los vidrios medievales analizados.

\subsection{Protocolo analítico.}

Se ha empleado una microsonda electrónica CAMECA SX50 con cuatro espectrómetros verticales, disponible en los Serveis Científico-Tècnics de la Universitat de Barcelona. Las 
condiciones analíticas han sido: un potencial de $20 \mathrm{kV}$ y una intensidad de corriente de $20 \mathrm{nA}$. Para evitar el pernicioso efecto de la migración de los elementos alcalinos (véase 8,9 y referencias en estos trabajos) el haz ha sido desenfocado $5 \mu \mathrm{m}$. En estas condiciones la conductividad eléctrica es buena y la reproductibilidad analítica satisfactoria. Cada uno de los vidrios analizados ha sido sondeado al menos en 20 puntos, distribuidos aleatoriamente a lo largo de la superficie de la lámina delgada, pero evitando las zonas inmediatamente asociadas a la superficie; esto se ha hecho tanto para evitar posibles zonas hidratadas y/o alteradas en ésta, analizadas mediante otros instrumentos, como para evitar valores analíticos espúreos por pérdida de continuidad eléctrica en la interfase vidrio-soporte u oquedades con la consiguiente pérdida de la superficie analítica especular en la muestra.

Los patrones empleados son silicatos y óxidos naturales y productos sintéticos de composición certificada, suministrados por las empresas P\&H Developments y Agar Cientific en dos bloques para microanálisis de 27 y 16 patrones respectivamente. Los patrones naturales empleados son ortosa ( $\mathrm{Si} ; \mathrm{Al}$; $\mathrm{O}$ en vidrios potásicos), albita ( $\mathrm{Na}$; $\mathrm{O}$ en vidrios sódicos), wollastonita $(\mathrm{Ca})$, especularita $(\mathrm{Fe})$, rodonita $(\mathrm{Mn})$, apatito $(\mathrm{P})$, calcopirita $(\mathrm{Cu})$ y galena $(\mathrm{Pb})$. Los patrones sintéticos ultrapuros de alta homogeinidad empleados son periclasa $(\mathrm{Mg})$, rutilo (Ti) y cloruro potásico $(\mathrm{Cl})$.

Todos los métodos que pretenden la cuantificación de la composición química de un sólido requieren el empleo de patrones de composición certificada. En algunos métodos los patrones requeridos deben ser lo más próximos posible en su composición global al sólido problema, para evitar efectos de interferencia de matriz. En el caso de los métodos basados en espectrometría de dispersión de longitud de onda el problema de la interferencia de matriz se minimiza (véase más adelante) de modo que se evita el empleo de patrones vítreos en la estandarización de las condiciones analíticas (por los problemas inherentes a la migración de los elementos alcalinos), empleándose diferentes patrones cristalinos que aporten singularmente uno o varios elementos a la estandarización. De este modo el empleo de vidrios de composición conocida queda relegado a su análisis como patrones internos en el curso del experimento, que es tal como se ha procedido en este trabajo.

La microsonda electrónica de Castaing permite explorar las muestras mediante espectometría de dispersión de longitudes de onda (WDS en el acrónimo anglosajón). En esencia se trata de aprovechar la ley de Braggs para filtrar la respuesta espectral de una muestra sometida a bombardeo electrónico mediante el empleo de cristales monocromáticos sintéticos que permiten únicamente que una estrecha franja de longitudes de onda emitidas por la muestra problema lleguen al detector.

En términos prácticos se obtiene en éste un espectro constituído por picos mucho más estrechos, lo que permite mejor resolución y límites de detección mucho menores que los del otro popular método analítico basado en haz de electrones, la espectrometría de rayos $X$ mediante dispersión de energías (EDS en el acrónimo anglosajón). La mejor resolución se traduce en general en la desaparición de las interferencias de las respuestas espectrales entre los diferentes elementos que se exploran (claramente presentes en el EDS) y los límites de detección de trabajo en condiciones de rutina instrumental pasan del 1-2 \% en peso a valores del orden del centenar de ppm. Otra diferencia práctica notable entre ambos métodos, normalmente relacionada con el diseño del instrumento al que sirven (en general un microscopio electrónico de barrido en el caso de la dispersión de energías) es la mayor estabilidad de la corriente de sonda durante la exploración de la muestra, lo que hace que la repetitividad de los análisis cuantitativos mediante espectrometría de dispersión de energías sea sensiblemente inferior a la obtenida mediante la microsonda electrónica que explota la espectrometría de dispersión de onda. Todo este conjunto de propiedades intrínsecas del método privilegian enormemente el empleo de la microsonda electrónica en el estudio de la química de sólidos cristalinos y vidrios respecto a la espectrometría de dispersión de energías asociada a un microscopio electrónico de barrido y por este motivo hemos empleado dicha técnica.

De todos modos las técnicas de espectrometría de dispersión de energías tienen algunas ventajas relativas referidas a las de dispersión de longitudes de onda, entre ellas la rapidez en la obtención de un espectro cualitativo. Por este motivo algunas microsondas de configuración moderna, entre ellas la que hemos empleado, incorporan un dispositivo de dispersión de energías que puede ser explotado ampliamente en la exploración preliminar en modo cualitativo o semicuantitativo de la muestra a analizar, lo que permite escoger el conjunto de elementos que se requiere analizar $\mathrm{y}$, en base a este conjunto, diseñar la secuencia de análisis elemental y el tipo de distribución de cristales monocromáticos y elementos en cada uno de los espectrómetros disponibles, con las consiguientes mejoras en rendimiento y velocidad en los análisis.

Los cristales analizadores empleados son los suministrados por la firma CAMECA con el instrumento, y concretamente se han empleado el LIF para $\mathrm{Fe}, \mathrm{Cu}$ y $\mathrm{Mn}$, TAP para $\mathrm{Si}$, $\mathrm{Al}$, Na y Mg, PET para $\mathrm{Ca}, \mathrm{K}, \mathrm{Ti}, \mathrm{Cl}$ y Pb. Además se ha determinado el $\mathrm{O}$ experimentalmente mediante el empleo de un cristal PC0.

La determinación del oxígeno merece un comentario aparte. Cuando se analiza una fase mineral con la microsonda electrónica normalmente se identifica previamente por vía petrográfica el mineral, se determinan los diferentes elementos que lo forman y aplicando un programa corrector que normalmente proporciona el fabricante se obtiene el $\mathrm{O}$ indirectamente, mediante el cálculo estequiométrico de los óxidos; en algunos casos a partir de este cálculo el programa suministrado por el fabricante proporciona incluso una estimación del agua contenida en un mineral dado (p.e., en el caso de las micas).

La determinación experimental del $\mathrm{O}$ permite obtener realmente los óxidos presentes (a partir de los diferentes elementos y el oxígeno determinados), contabilizar el $\mathrm{O}$ residual $\mathrm{y}$, mediante estequiometría (calculando el $\mathrm{H}$ requerido) obtener el porcentaje en agua. Esta técnica no es trivial, ya que se requiere una configuración instrumental especial que no todas las microsondas poseen ( $\mathrm{y}$ a la que afortunadamente hemos tenido acceso) y la medición del oxígeno en diferentes patrones según el material a analizar sea sódico o potásico (tal como se ha indicado más arriba), ya que en el caso del oxígeno sí que existen elementos de interferencia muy perniciosos en el resultado final del análisis si se escoge un patrón inadecuado. Para realizar este tipo de análisis se ha diseñado una rutina analítica específica para vidrios (7), que posteriormente se ha desdoblado en dos para el estudio de vidrios dominantemente sódicos o potásicos, respectivamente.

Los resultados analíticos que se exponen (tabla 1) representan el promedio de los puntos sondeados en cada vidrio (n $\geq 20$ ); de hecho la homogeinidad en el seno de cada vidrio es muy remarcable y las diferencias apreciables son en la totalidad de los casos inferiores al ruido instrumental. 
TABla 1: COMPOSición QUímiCA (ANÁLISIS MEDIANTE MICROSONDA ELECTRÓNICA) DE LOS VIDRIOS DE LA IGLESIA DEL MONESTIR DE PEDRALBES ANALIZADOS.

\begin{tabular}{|l|l|l|l|l|l|l|l|l|l|l|}
\hline & Blanco & Azul & $\begin{array}{l}\text { Azul } \\
\text { con } \\
\text { grisalla }\end{array}$ & Rosado & Rosado & Amarillo & $\begin{array}{l}\text { verde } \\
\text { oscuro } \\
\text { con } \\
\text { grisallida }\end{array}$ & $\begin{array}{l}\text { verde } \\
\text { oscuro } \\
\text { con } \\
\text { grisalla }\end{array}$ & $\begin{array}{l}\text { verde } \\
\text { claro }\end{array}$ & $\begin{array}{l}\text { Rojo } \\
\text { plaqué }\end{array}$ \\
\hline $\mathrm{Fe} 2 \mathrm{O} 3$ & 0.64 & 1.60 & 1.08 & 0.67 & 0.76 & 0.76 & 1.19 & 1.24 & 0.65 & 0.48 \\
\hline $\mathrm{Na} 2 \mathrm{O}$ & 17.90 & 16.19 & 19.02 & 17.32 & 16.71 & 17.38 & 13.06 & 13.45 & 12.09 & 0.48 \\
\hline $\mathrm{K} 2 \mathrm{O}$ & 2.64 & 3.25 & 2.95 & 2.89 & 2.88 & 2.78 & 4.08 & 4.07 & 2.83 & 16.84 \\
\hline $\mathrm{MnO}$ & 1.01 & 1.76 & 0.03 & 1.46 & 1.51 & 0.15 & 1.75 & 1.71 & 0.11 & 0.90 \\
\hline $\mathrm{SiO} 2$ & 62.42 & 59.95 & 61.82 & 62.14 & 63.22 & 64.89 & 58.89 & 60.06 & 65.78 & 53.90 \\
\hline $\mathrm{A} 2 \mathrm{O} 3$ & 4.04 & 3.86 & 1.97 & 3.45 & 3.74 & 1.98 & 3.67 & 3.64 & 0.86 & 2.28 \\
\hline $\mathrm{MgO}$ & 2.08 & 1.87 & 1.99 & 1.88 & 2.10 & 3.69 & 1.81 & 1.81 & 2.28 & 3.48 \\
\hline $\mathrm{CaO}$ & 6.57 & 7.18 & 7.93 & 6.51 & 6.48 & 6.06 & 9.08 & 9.06 & 8.41 & 14.08 \\
\hline $\mathrm{TiO} 2$ & 0.21 & 0.22 & 0.11 & 0.19 & 0.19 & 0.08 & 0.22 & 0.22 & 0.01 & 0.17 \\
\hline $\mathrm{CuO}$ & 0.05 & 0.005 & 0.40 & 0.02 & 0.04 & 0.008 & 1.68 & 2.27 & 5.90 & 0.03 \\
\hline $\mathrm{Cl}$ & 1.40 & 1.06 & 1.37 & 1.29 & 1.45 & 1.94 & 1.03 & 0.75 & 0.87 & 0.46 \\
\hline $\mathrm{P} 2 \mathrm{O} 5$ & 0.87 & 0.16 & 0.88 & 0.08 & 0.02 & 0.05 & 0.61 & 1.03 & 0.37 & 4.33 \\
\hline $\mathrm{H} 2 \mathrm{O}$ & 0.40 & 3.08 & 0.43 & 2.21 & 1.68 & 0.14 & 2.91 & 1.13 & 1.13 & 2.25 \\
\hline $\mathrm{Total}$ & 100.32 & 100.23 & 100.03 & 100.18 & 100.84 & 99.96 & 100.04 & 100.50 & 101.31 & 99.74 \\
\hline
\end{tabular}

\subsection{Características químicas de los vidrios}

Los resultados analíticos que exponemos quedan resumidos en la tabla 1. Todos los vidrios, con la excepción del plaqué rojo (para el que no es válida obviamente la explicación que sigue), se caracterizan por ser sódicos, en un rango de composición del orden de 13-19 \% $\mathrm{Na}_{2} \mathrm{O}$. Los contenidos en $\mathrm{CaO}$ son relativamente bajos, entre el 6 y el $9 \%$ en peso (14\% en el caso del vidrio potásico rojo). Los contenidos en sílice oscilan entre el 59 y el $63 \%$ (54\% en el vidrio rojo), mientras que la suma de los óxidos de los elementos considerados vitrificantes $\left(\mathrm{SiO}_{2}+\right.$ $\mathrm{Al}_{2} \mathrm{O}_{3}+\mathrm{P}_{2} \mathrm{O}_{5}$ ) queda siempre por encima del $60 \%$, lo que está en buena concordancia con su estabilidad; por el contrario la suma de los óxidos alcalinos queda siempre ampliamente por encima del $17 \%$ y, en el caso de algunos de los sódicos, por encima del $20 \%$, lo que sería un indicio de una potencial inestabilidad (10). Cabe señalar en todo caso que el contenido en elementos alcalinos del vidrio rojo es menor que en los sódicos, o si se prefiere, que el vidrio potásico es moderadamente alcalino si se compara con otros vidrios europeos $(1 ; 2,11$, p. 114; Müller in 12, p.89; 13, etc.) . El MgO presenta valores inferiores al $2 \%$, y el fósforo expresado en forma de pentóxido es inferior al $1 \%$. Finalmente los contenidos de $\mathrm{MnO}$ en la mayor parte de estos vidrios es inferior al 2\%, y los contenidos de plomo son muy bajos (con la evidente excepción de las grisallas, que no aparecen recogidas en la tabla 1), por lo que podemos considerar a este elemento como un minoritario accidental en estos vidrios sin protagonismo ni por lo que se refiere a su estabilidad ni al color.

Por lo que se refiere a la gama cromática, los colores básicos en el vitral estudiado son azul oscuro, verde claro, verde oscuro, rosado, amarillo y, naturalmente, blanco. El vidrio rojo es minoritario y su uso se ha restringido sólo a sectores nobles de la iconografía.

El vidrio azul presenta una composición comparable a la del vidrio blanco, con la diferencia que contiene como colorante óxido de cobalto en cantidades inferiores al $0.5 \%$ (determinado mediante FRX). El vidrio verde, que tiene un espectro de componentes presentes en traza sensiblemente más complica- do que otros vidrios, por lo que se refiere a sus componentes mayoritarios se distingue en sus dos modalidades (claro y oscuro) principalmente por la presencia de un colorante muy evidente en forma de un mínimo de 1.5-2 \% de $\mathrm{CuO}$, aproximadamente.

La composición del vidrio rosado es extraordinariamente parecida a la del vidrio blanco, con la única y pequeña diferencia de un incremento del orden del $50 \%$ en la cantidad de $\mathrm{MnO}$, que de todas maneras apenas llega al $1.5 \%$. El porcentaje de hierro presente es muy bajo ( $0.75 \%$ aproximadamente) en ambos casos.

Los vidrios amarillos presentan bastante más $\mathrm{MgO}$ que los blancos (un 70-75 \% más), un $10 \%$ menos de $\mathrm{CaO}$, un $50 \%$ de la alúmina que presentan los blancos, una sexta parte del manganeso y un $30 \%$ menos de titanio. Además cabe señalar que los contenidos del los vidrios amarillos por lo que se refiere a la sílice, el sodio y el cloro son de los más elevados, entre todos los analizados.

Por lo que se refiere a las grisallas, presentan una composición poco relevante, caracterizada por la presencia de calcio, hierro y plomo (que fue empleado como fundente en la vitrificación en horno de las pinturas). Su estado de conservación es bueno, lo que no es extraño si consideramos que el del vidrio también lo es, lo que elimina de entrada todos los problemas de adherencia, y además indica de manera indirecta que las condiciones ambientales han sido favorables para su conservación.

\section{DISCUSIÓN DE LOS RESULTADOS}

El conjunto de los vidrios, con la excepción del rojo "plaqué", presenta unas características composicionales que permiten calificarlos como químicamente estables, tanto por el hecho de ser sódicos como por sus elevados porcentajes de sílice; y, dentro del contexto europeo del siglo XIV, como relativamente raros, ya que es bien conocida la predominancia de vidrios potásicos $(1,12$, etc.). 
Los elementos alcalinos son introducidos en la mezcla que se lleva al horno para conseguir bajar la temperatura de fabricación del vidrio, y en general durante el periodo medieval este tipo de elementos eran introducidos en forma de cenizas de vegetales $(12,14$, etc.). Hay que matizar que la información disponible sobre los vidrios sódicos es aún más fragmentaria que la referida a los potásicos; por ejemplo, la conocida receta del monje Theophilus (15) recogida a inicios del siglo XII es de muy improbable aplicación, incluso por lo que se refiere a la dosificación, a los vidrios sódicos.

En principio el vidrio de Pedralbes es de procedencia mediterránea y no es descabellado pensar en un taller local (16, p 34; 15), y de hecho existe alguna información indirecta que indica la producción de vidrio plano arquitectónico en Cataluña hacia 1189 (17, p. 44). El sodio procede posiblemente de cenizas de plantas crecidas en zonas salobres, pero los contenidos en sodio de estas cenizas no tiene por qué ser comparables al del potasio presente en la ceniza de haya, consecuentemente en el caso que nos ocupa la receta de Theophilus sólo tiene que ser tomada como indicativa. Los bajos contenidos en calcio indican que este elemento no estaba presente en cantidades importantes en las cenizas vegetales utilizadas, e incluso no cabe excluir su incorporación al horno en forma mineral. Se verá más adelante que esto puede inferirse de la composición del vidrio amarillo. Los contenidos de $\mathrm{MgO}$ y de $\mathrm{MnO}$ no son directamente relacionables con las variaciones en el contenido en sodio, siendo la interpretación más sencilla que estos elementos no tienen porqué estar exclusivamente ligados en su introducción en la mezcla base del vidrio a partir de cenizas vegetales, o en todo caso a la llegada del sodio a la pasta base del vidrio.

Las informaciones históricas sobre la incorporación del color a los vidrios son más que fragmentarias; de hecho en las diferentes copias conservadas del citado tratado de Theophilus los capítulos concernientes han sido eliminados (15), quedando únicamente aquellas referencias de coloración atribuibles (a la luz de los conocimientos actuales) a variaciones en las condiciones redox en el horno. Por otra parte, existe todo un cuerpo de doctrina consolidado sobre el papel de los elementos de transición como elementos colorantes en un vidrio (véase p.e. $16,18,19)$.

Así, el vidrio rosado de Pedralbes no parece interpretable como el producto de una más prolongada permanencia del fundido en el seno de un horno dotado de atmósfera oxidante, en el sentido expresado implícitamente por Teophilus, sino más bien como el producto de una adición de manganeso a la mezcla base del vidrio blanco, o bien por una casual administración de este exceso de Mn (p.e. en las cenizas) y una prolongada residencia del fundido en el seno de un horno oxidante, de manera que se aprovecharan los vidrios virados a color rosa carne, y el resto sencillamente como blancos.

Los vidrios azules presentan un colorante clásico en forma de Co (determinado en modo EDS en la MSE), y en este sentido no aportan grandes novedades aparte de la confirmación del empleo de una sal de este elemento en su elaboración. Los vidrios verdes presentan un colorante claro, el Cu; (20) in (12) ha señalado que este color se producía por la presencia conjunta de óxido cúprico y óxido férrico en vidrios fundidos en condiciones oxidantes, y es significativo al respecto que el contenido en hierro del vidrio verde oscuro es sensiblemente superior al resto (excepción hecha del vidrio azul), casi el doble. Si comparamos los vidrios de color verde claro respecto al oscuro se observa un mayor contenido en $\mathrm{Cu}$ (x2-2.5 el contenido del vidrio oscuro), 2/3 del contenido en $\mathrm{K}$, algo menos de $\mathrm{Na}$, $\mathrm{y}$ aproximadamente la mitad del $\mathrm{Fe}$, es decir en el rango compo- sicional del resto de los vidrios sódicos. Estos datos han de interpretarse como una confirmación de que el elemento colorante es el $\mathrm{Cu}$, y que su mayor intensidad evidentemente se debe a la asociación con un incremento en la cantidad de hierro adicionado; la dosificación parece demasiado precisa (más cobre sin hierro verde claro, menos cobre con hierro verde oscuro) para ser fruto del azar.

Los vidrios amarillos son bastante semejantes en su composición a los blancos; las diferencias en alúmina, titanio, e incluso quizás en el calcio pueden ser atribuídos a un menor contenido en arcillas en las arenas silíceas empleadas. Es decir, podemos interpretar que las arenas cuarcíferas fueron limpiadas o escogidas más cuidadosamente en este caso. El color amarillo no puede corresponder ni al hierro (siempre en valores bajos, $0.75 \%$, y del mismo orden que en el vidrio blanco) ni al manganeso, que también ha sido eliminado de la materia prima o no añadido a la mezcla del horno. Parece por lo tanto claro que el fabricante del vidrio incrementó su cuidado en eliminar elementos nocivos (hierro y manganeso) de cara a la obtención controlada de color, y al mismo tiempo añadió magnesio, elemento que en general confiere color verde (por ej., en muchos silicatos naturales como forsterita, clorita, crisotilo), y amarillo en el caso de un material transparente como el vidrio. No disponemos de criterios concluyentes respecto a de qué manera se incorporó el magnesio a la pasta base del vidrio, pero sea mineral o vegetal tiene que ser muy específica y conllevar la llegada a ésta de un contenido adicional de cloro.

El vidrio rojo es sensiblemente diferente del resto, tanto por su composición como por la tecnología de fabricación ("plaqué"); es muy parecido a la norma del vidrio europeo, y en particular centroeuropeo, del siglo XIV. Se trata de un vidrio potásico, con más de un $16 \%$ de $\mathrm{K}_{2} \mathrm{O}$, más de un $14 \%$ de $\mathrm{CaO}$, y más de un $4 \%$ de $\mathrm{P}_{2} \mathrm{O}_{5}$. El Na $2 \mathrm{O}$ no llega al $0.5 \%$. Además se trata de un vidrio tecnológicamente más avanzado, no tanto por lo que se refiere al menor espesor y menor presencia de burbujas (atribuibles al mayor intervalo térmico de estabilidad de trabajo del vidrio potásico (21), sino por la aplicación de color en capa superficial, caracterizada por la presencia de $\mathrm{Cu}(0.4-0.5 \%)$ únicamente en el estrato coloreado. La tecnología de esmaltado del estrato coloreado ha sido satisfactoriamente explicada (22), si bien el vidrio rojo de Pedralbes presenta diferentes laminillas de color rojo intercaladas con el vidrio blanco y superpuesta una capa protectora de vidrio incoloro, hecho que nos hace pensar en que el proceso de fabricación debió ser otro.

En conjunto la composición, factura, escasez y ubicación en motivos iconográficos importantes dentro de la vidriera son factores que abogan a favor de un origen diferente de este vidrio. Posiblemente se trate de un vidrio centroeuropeo importado; hay que esperar que el análisis de documentación de la época de realización de las vidrieras confirme esta hipótesis.

\section{CONCLUSIONES}

El estudio desarrollado ha permitido la caracterización de los vidrios de la gama de color existente en la vidriera de Sant Pere y Sant Jaume (O-II-9) de la iglesia gótica del Real Monasterio de Pedralbes, correspondientes a la primera mitad del siglo XIV, y preservados en su gran mayoría con un emplomado original. Aunque los resultados obtenidos no son necesariamente representativos de la totalidad de la vidriera del presbiterio de la citada iglesia, destaca notablemente que se trate de vidrios sódicos de influencia mediterránea, frente a los potásicos predominantes en ese momento en toda Europa, si nos atenemos a la información disponible. Resulta evidente por 
tanto que estos vidrios sódicos deberían ser contrastados con otros coetáneos, y en particular italianos, para clarificar un poco más los tipos de fabricación de vidrio plano en Europa, al menos durante los siglos XI-XV. Estos vidrios son de factura relativamente primitiva, en particular si comparamos con el único potásico localizado hasta el momento (rojo rubí plaqué), tecnológicamente mucho más avanzado, lo que nos hace pensar en un aprovisionamiento diversificado durante el proceso de la obra, e incluso en la presencia de un taller local para la producción del vidrio sódico.

La metodología analítica empleada ha permitido contrastar la eficacia de la microsonda electrónica en la caracterización de los vidrios y, en muchos casos, de los pigmentos originales empleados en su coloración. En términos prácticos esto implica que se requiere el sacrificio de pequeñísimas cantidades de vidrio para la caracterización de toda la gama de color de la vidriera, es decir que en términos prácticos se trata de una metodología analítica respetuosa y no invasiva para con la vidriera. La gama composicional hallada permite extraer conclusiones sobre el proceso de fabricación de los vidrios de diferentes colores, partiendo del vidrio blanco como clave de lectura en la génesis de los colores.

Los vidrios sódicos presentan un estado de conservación muy bueno, mientras que el potásico presenta evidencias de una alteración moderada pero marcadamente evidente. En términos de conservación y restauración esto implica mucha economía de recursos a emplear en el caso de vidrios sódicos: e incluso en el caso del vidrio potásico las diferencias composicionales inciden directamente sobre la resistencia de éste a los procesos de corrosión química y a los ataques biológicos. Consecuentemente si se realiza un mapeo del tipo de vidrio presente, un muestreo de la gama de color presente y una analítica de caracterización del vidrio del tipo de la realizada en este trabajo al inicio de un proceso de intervención no sólo se obtienen valiosas informaciones de tipo histórico (tecnología de fabricación, materias primas y pigmentos empleados) sino que además se pueden establecer con más precisión los criterios de restauración y el presupuesto requerido en cada intervención, cosa que debiera interesar a las administraciones y propietarios implicados en el proceso de restauración, al tiempo que a los profesionales.

En otro orden de cosas, el escaso desarrollo de la alteración, incluso en los vidrios potásicos, indica que tanto el clima mediterráneo, como la situación del monasterio relativamente alejado de la línea de costa y del centro histórico de la ciudad, e igualmente el hecho de la que iglesia haya sido relativamente poco frecuentada (si comparamos con algunas catedrales europeas) con la consiguiente escasa generación de condensaciones en el interior, han permitido una buena conservación del vidrio arquitectónico a pesar de su metaestabilidad.

La detección del proceso de alteración de los vidrios potásicos de Pedralbes en un estadio muy incipiente puede permitir por otra parte en estudios sucesivos (en curso) aclarar cómo comienzan a desarrollarse los procesos de corrosión y aportar nuevas luces sobre la coexistencia y retroalimentación de los fenómenos puramente químicos y los biológicos que se desarrollan sobre la superficie del vidrio.

\section{AGRADECIMIENTOS}

Este estudio ha sido financiado por el Ajuntament de Barcelona mediante el proyecto 3338 de la Fundació Bosch i Gimpera (UB). La caracterización geoquímica de los vidrios se ha llevado a cabo en los Serveis Científico-Tècnics de la
Universitat de Barcelona (SCT-UB), y la preparación de las muestras se ha llevado a cabo en los laboratorios del Dept. Geoquímica, Petrologia i Prospecció Geològica y en el Servei de Làmina Prima (UB); los autores agradecen todo el personal técnico y científico involucrado, y en particular a J.García Veigas, E.Seguí y J.M.Socias la asistencia e ideas aportadas durante el desarrollo de la parte experimental. La restauración de los vitrales ha sido posible gracias a la inestimable colaboración de la Comunidad de Clarisas del Monestir de Pedralbes, dentro de un programa financiado igualmente por el Ajuntament de Barcelona (director del proyecto J.M.Julià). Los autores desean agradecer a todos los implicados en el proyecto, y en especial al equipo de restauración (coordinado por M.Pugès) la colaboración en diferentes momentos de desarrollo del estudio. Las fotografías han sido realizadas por Josep Gri y aparecen por gentileza del Ajuntament de Barcelona.

\section{BIBLIOGRAFÍA}

1. R.H.Brill "Chemical analysis of early glasses". Vol. 2, The Corning Museum of Glass, Corning, New York, 335 pp1999,

2. R.H.Brill “Composición química de algunos vidrios de la Catedral de León”, 114-131, In "Conservación de vidrieras históricas. Proceedings of an international seminar held in Santander, Spain, July 4-8, 1994", M.A.Corzo \& N.Valentín (Eds) The J.Paul Getty Trust, USA, 1997.

3. J.Ainaud De Lasarte, A.A.Mundó, J.Vila-Grau, M.A.Escudero Ribot, S.Canyellas, A.Vila Delclós. "Els vitralls de la Catedral de Barcelona i del Monestir de Pedralbes". Corpus Vitrearum Medi Aevi, Institut d'Estudis Catalans, Barcelona, 394 pp, (1997).

4. G.Lofgren. "Experimentally produced devitrification textures in natural rhyolitic glass". Geol.Soc.Am.Bull. 82: 111-124 (1971).

5. S.E.Swanson, M.T.Naney, H.R.Westrich, J.C.Eichelberber "Crystallization history of obsidian dome, Inyo Domes, California". BullVolcanol. 51: 161-176 (1989).

6. O.Gedeon, K.Jurek, V.Hulínsky . "Fast migration of alkali ions in glass irradiated by electrons" J.Non-Cryst.Solids 246:1-8, (1999).

7. N.Díaz., J.García Veigas, D.Gimeno. “Desarrollo de una metodología de análisis de vidrios volcánicos ácidos, y sus equivalentes desvitrificados, por microsonda electrónica". Bol.Soc.Esp. Mineralogía 21 (A): 74-75 (1998).

8. C.H.Nielsen, H.Sigurdsson. "Quantitative methods for electron microprobe analysis of sodium in natural and synthetic glasses". American Mineralogist, 66: 547-552, (1981).

9. O.Gedeon, V.Hulínsky, K.Jurek. "Microanalysis of glass containing alkali ions". Mikrochim.Acta, 132: 505-510, Springer Verlag, (2000).

10. J.M.Fernández Navarro."Procesos de alteración de las vidrieras medievales. Estudio y tratamientos de protección", Materiales de Construcción, 46 (242-243): 5-25 (1996).

11. A.Corallini, V.Bertuzzi. "Il restauro delle vetrate" 253 pp, Nardini Editore, Fiesole (FI, Italia), 1994

12. J.M.Fernández Navarro. "Constitución química de las vidrieras y métodos para su análisis y para el estudio de sus alteraciones", 85-113, In "Conservación de vidrieras históricas. Proceedings of an international seminar held in Santander, Spain, July 48, 1994", M.A.Corzo \& N.Valentín (Eds) The J.Paul Getty Trust, USA, 1997.

13. M.R.Schreiner, I.Prohaska, J.Rendl, C.Weigel. "Leaching studies of potash-lime-silica glass with medieval glass composition", 72-83, In "The conservation of glass and ceramics", N.H.Tennent, James \& James (Science Publishers), Ltd, London, 1999.

14. R.Newton, S.Davison. "Conservation of glass". 318 pp, Butterworth-Heineman, Oxford, U.K., 1989.

15. J.G.Hawthorne, C.S.Smith. “Theophilus: On Divers Arts. The foremost medieval treatise on painting, glassmaking and metalwork" (traducción del latín con introducción y notas técnicas de los autores). 216 pp, Dover Publications Inc., New York, 1979.

16. J.M.Fernández Navarro. "El vidrio. Constitución, fabricación, propiedades", 667 pp, CSIC, Madrid, 1985.

17. V.Nieto Alcaide. “La vidriera española”. 416 pp, Nerea, Madrid, 1998.

18. E.Damour . "Cours de verrerie. IIIème partie. Le refroidissement du verre. Le travail du verre. Propietés physiques à froid". 273 pp, Lib. Polytechmique Ch.Béranger, Ed., Paris, 1936

19. P.Bary, J.Herbert. “La verrerie" 270 pp Ed. Dunod, Paris, 1941

20. C.D.Vassas. "Étude chimique, thermographique et physique de verres de vitraux du Moyen Age". In $9^{\text {th }}$ International Congress on Glass, Versailles, France, 1971, Institut du Verre, Paris, 1972.

21. J.M.Fernández Navarro. “Características de las materias primas para la fusión de diferentes tipos de vidrios". Bol.Soc.Esp.Cerám.Vidrio, 28 (6): 449-459 (1989).

22. J.M.Fernández Navarro, A. La Iglesia. "Estudio de la coloración roja y amarilla de vidrios de la Catedral de Toledo". Bol.Soc.Esp.Cerám.Vidrio, 33 (6): 333-336, (1994).

Recibido: 16.11 .00

Aceptado: 30.10 .01 